\title{
Supporting Information for The Role of Scale Wettability on Rain-Harvesting Behavior in a Desert-Dwelling Rattlesnake
}

Akshay Phadnis, ${ }^{1}$ Kenneth C. Manning, ${ }^{1}$ Gordon W. Schuett,,${ }^{2,3}$ and Konrad Rykaczewski ${ }^{1, *}$

1. School for Engineering of Matter, Transport and Energy, Arizona State University,

Tempe, AZ, USA

2. Chiricahua Desert Museum, Rodeo, NM, USA

3. Department of Biology and Neuroscience Institute, Georgia State University, Atlanta,

GA, USA

\section{S1. Imaging of TPCL during droplet evaporation from P.catenifer scales}
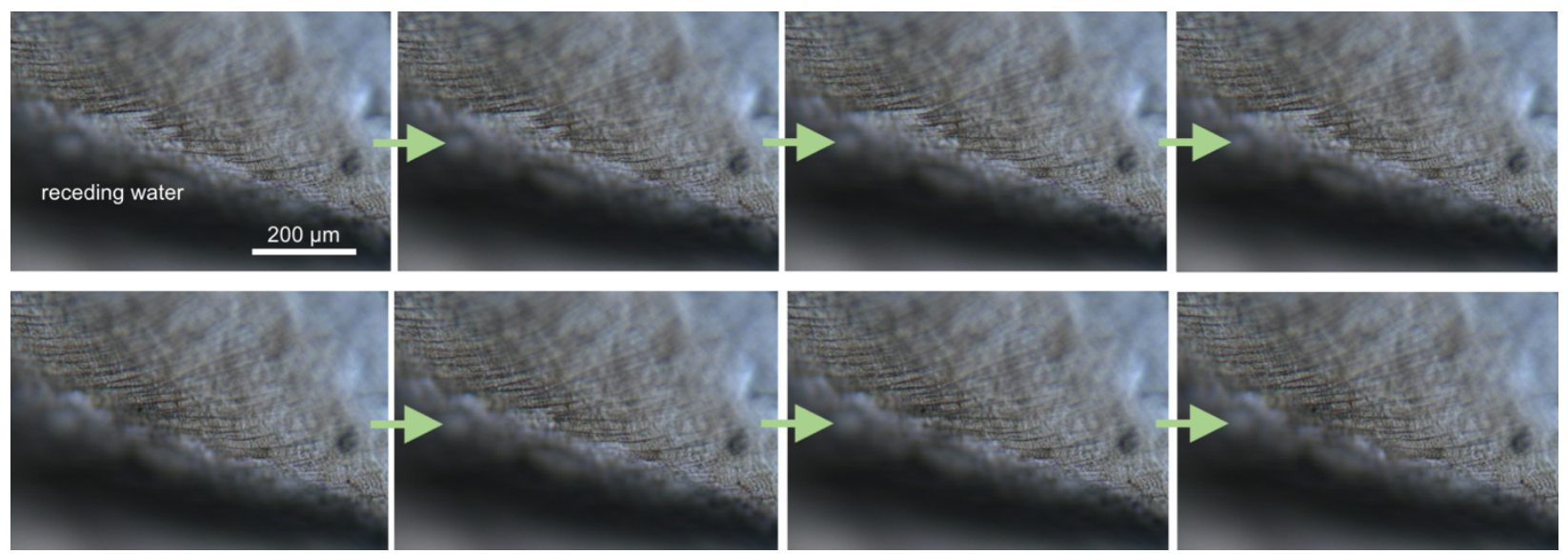

Figure S1. Sequence of light microscopy images showing edge of a drying droplet on the scales of the P.catenifer.

\section{S2. Additional imaging of water droplets on C.atrox scales}

To confirm the water penetration in nanochannels we evaluated the wetting behavior of pigmented droplets (fire engine red, KEDA dye) on a dry shed skin of C. atrox (see Figure S1). Since the shed skin is transparent, we were able to directly image the droplet-surface interface through the skin (i.e. from the opposite side). A microscopic zoomed-in view of this image is also shown in Figure S1. If air is trapped underneath water droplets, such as on superhydrophobic 
plants, a smooth air-liquid interface that is highly reflective forms. ${ }^{1}$ The absence of reflective spots in the microscopic image implies that there are no air pockets captured at the water-surface interface and confirms full penetration of the liquid into the nanochannels. Thus, the overall effect of this texture results in sticky Wenzel state (droplets do not shed even when surface is turned upside down) with moderately high apparent contact angle and high contact angle hysteresis.
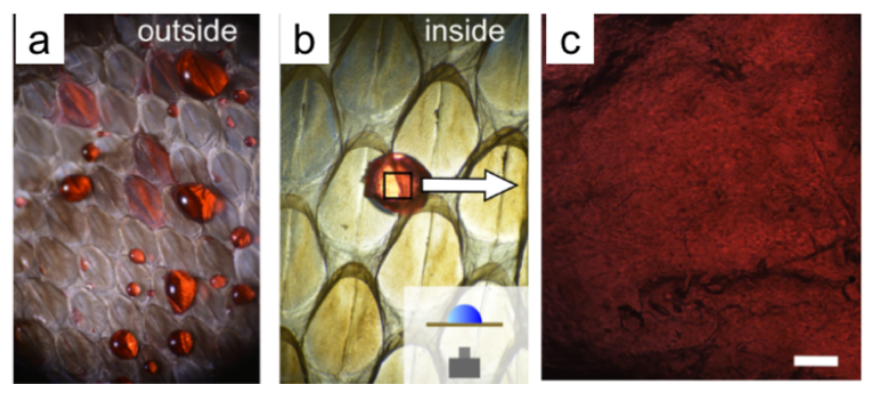

Figure S2. (a) Image of several dyed water droplets sticking to the shedded dry skin of C. atrox (outer side of the skin), (b) Image of a sessile droplets on the shedded dry skin (outer side) of $C$. atrox taken rom the other side of the skin (inner side), (c) microscope image of droplet in (b). Scale bar $=100 \mu \mathrm{m}$.

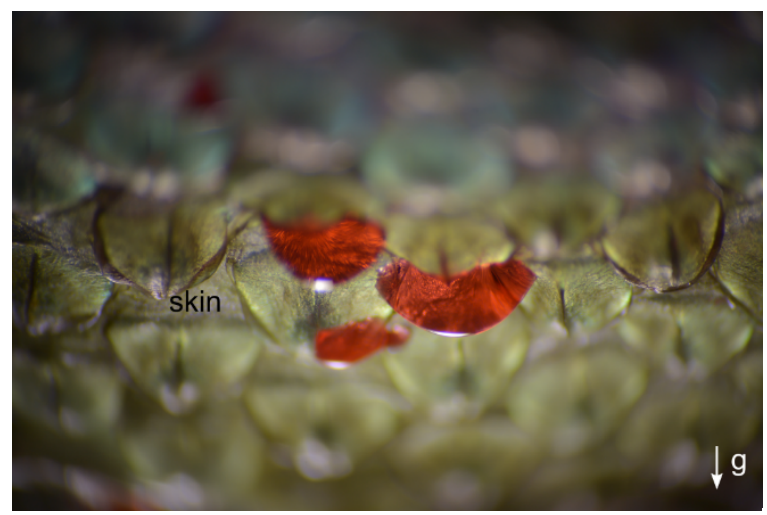

Figure S3. An image of an inverted shed skin of C. atrox with a few water droplets holding on to it. 


\section{S3. Comparison of topography of dorsal and ventral scales of C.atrox}
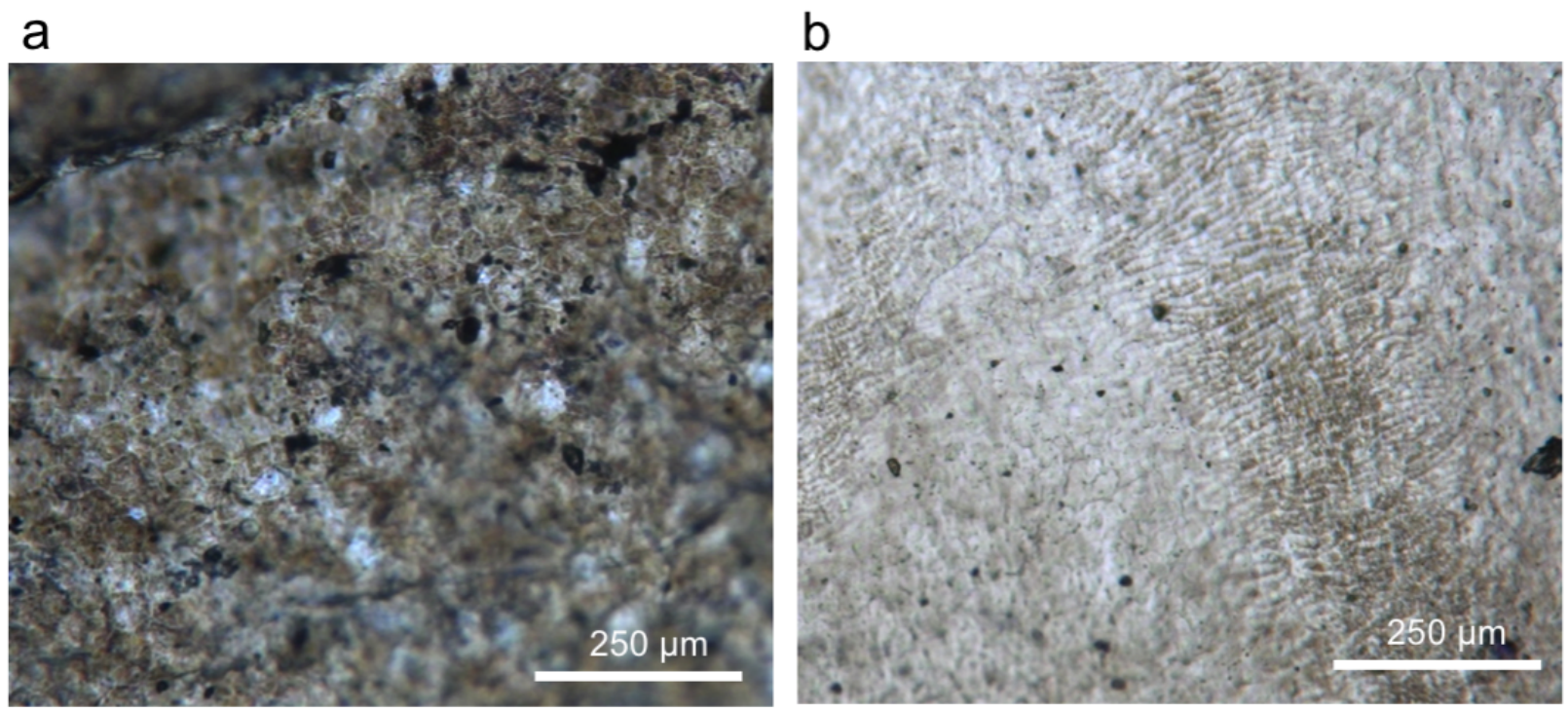

Figure S4. Optical image of (a) dorsal and (b) ventral scale of shed skin of C. atrox. The distinct four to five sided regions are visible in (a) but not in (b) where more pronounced line-like microscopic features are present.

\section{S4. Scematic of the droplet-impact setup}

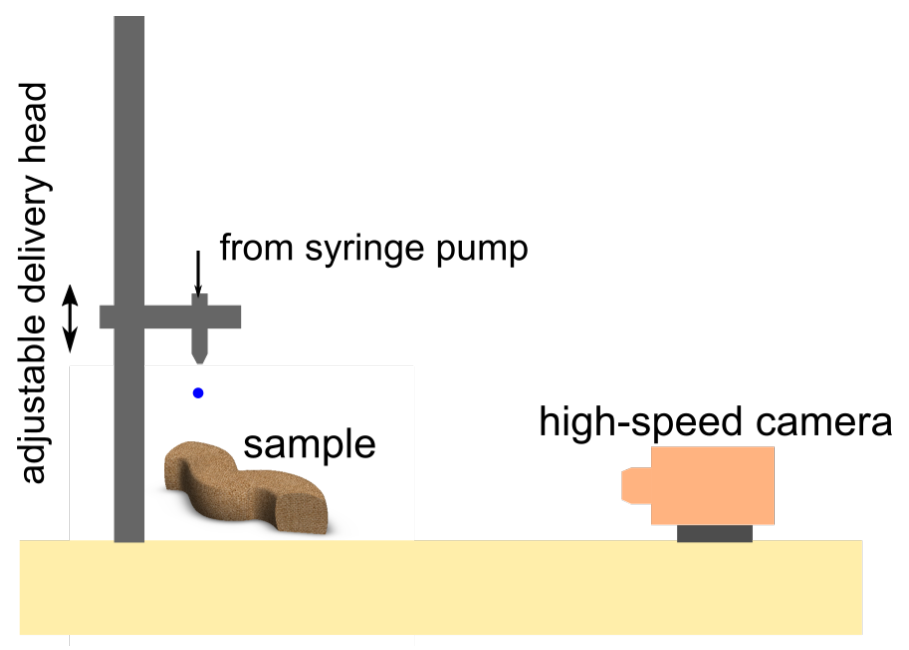

Figure S5. Schematic of the setup used for droplet-impact experiments 